\title{
On irreducible components of a Weierstrass-type variety
}

\author{
by Romuald A. JANIK (Kraków)
}

\begin{abstract}
We give a characterization of the irreducible components of a Weierstrasstype ( $W$-type) analytic (resp. algebraic, Nash) variety in terms of the orbits of a Galois group associated in a natural way to this variety. Since every irreducible variety of pure dimension is (locally) a component of a $W$-type variety, this description may be applied to any such variety.
\end{abstract}

1. Introduction. This work grew out of an attempt to provide an algebraic description of analytic varieties of constant dimension. We study Weierstrass type varieties introduced by Whitney in [6]. Since any analytic set of constant dimension is a sum of irreducible components of a $W$-type variety (see [6], p. 81), we can consider only the irreducible components of a Weierstrass-type set.

The main aim of this paper is to characterize the irreducible components of a $W$-type variety in terms of an action of a Galois group associated in a natural way with the given variety.

Let $U$ be an open connected subset in $\mathbb{C}^{n}$. We denote by $R$ one of the following rings:

- $\mathbb{C}[u]=$ ring of polynomials in $n$ variables,

- $\mathcal{O}(U)=$ ring of holomorphic functions on $U$,

- $\mathcal{N}(U)=$ ring of Nash functions on $U$.

The ring $\mathcal{N}(U)$ is the algebraic closure of $\mathbb{C}[u]$ in $\mathcal{O}(U)$ (for further properties of Nash functions cf. [5]).

Let $W$ be a $W$-type (Weierstrass type) $n$-dimensional variety in a connected open set $U \times \mathbb{C}^{k} \subset \mathbb{C}^{n+k}$ :

$$
W=\left\{\left(u, z_{1}, \ldots, z_{k}\right) \in U \times \mathbb{C}^{k} \mid p_{i}(u)\left(z_{i}\right)=0, i=1, \ldots, k\right\}
$$

1991 Mathematics Subject Classification: Primary 14E20, 32C25; Secondary 32B15, $12 \mathrm{~F} 10$.

Key words and phrases: branched covering, Weierstrass-type variety, Galois theory. 
where

$$
p_{i}(u)(z)=z^{n_{i}}+\sum_{j=0}^{n_{i}-1} a_{j}^{i} z^{j} \in R[z], a_{j}^{i} \in R .
$$

Let $K$ be the field of fractions of the ring $R$, and $L$ be a common splitting field over $K$ of the defining polynomials $p_{1}, \ldots, p_{k}$ of $W$. Then $L / K$ is a Galois extension. Let $X_{i} \subset L$ be the zero-set of the polynomial $p_{i}$ in $L$. The Galois group $\operatorname{Gal}(L / K)$ acts in a natural way on $X_{1} \times \ldots \times X_{k}$. We prove that the orbits of this action are in 1:1 correspondence with the irreducible components of $W$ (Theorem 6.1).

In Section 2 we introduce some notation and state a few basic facts from Galois theory. In Section 3 we construct a covering space $\operatorname{Hom}(L, \mathcal{M})$ of $U^{\prime}:=U \backslash\{$ branching locus of $W\}$, encoding the algebraic and topological structure of the problem. We investigate its relations with $W$, which will be exploited in the proof of the irreducibility of the variety associated with an orbit of $\operatorname{Gal}(L / K)$. In Section 4 the properties of $\operatorname{Hom}(L, \mathcal{M})$ are used to construct a homomorphism from the fundamental group $\pi_{1}\left(U^{\prime}\right)$ to the Galois group $\operatorname{Gal}(L / K)$.

In Section 5, certain finite subsets of $L^{k}$ are shown to correspond to irreducible components of $W$. Finally, in Section 6, we state and prove our main result. The proof is based on the observation that every irreducible component of $W$ can be obtained by the construction described in Section 5 .

2. Algebraic preliminaries. We begin with two results from Galois theory.

Lemma 2.1 ([2], p. 42). Let $K$ be an algebraic extension of the field $k$ contained in the algebraic closure $\bar{k}$ of $k$. Then the following statements are equivalent:

1. The field extension $K / k$ is normal.

2. Every $k$-homomorphism $\sigma: K \rightarrow \bar{k}$ is onto $K$.

Lemma 2.2. Let $L / K$ be an algebraic field extension, and $K \subset \Omega$ an arbitrary field extension. Then there is a K-homomorphism $\sigma: L \rightarrow \bar{\Omega}$.

Let $u$ be a point in $U$. We shall use the following notation:

- $\mathcal{O}_{u}=$ ring of germs of holomorphic functions at $u$,

- $\mathcal{M}_{u}=$ field of fractions of the ring $\mathcal{O}_{u}$,

- $\mathcal{M}(U)=$ field of fractions of the ring $\mathcal{O}(U)$,

- $\widetilde{\mathcal{N}}(U)=$ field of fractions of the ring $\mathcal{N}(U)$ of Nash functions.

Let $\delta_{i} \in \mathcal{O}(U)$ be the discriminant of the polynomial $p_{i}(z) \in \mathcal{O}(U)[z]$ (for the definition and basic properties of the discriminant see e.g. [3], p. 25). Let $\Delta$ be the discriminant variety $\Delta:=\left\{u \in U: \delta_{1}(u) \cdot \ldots \delta_{k}(u)=0\right\}$. We shall 
denote its complement in $U$ by $U^{\prime}$. Then $U^{\prime}$ is the biggest open set such that $\pi:\left(U^{\prime} \times \mathbb{C}^{k}\right) \cap W \ni(u, z) \mapsto u \in U^{\prime}$ is an unbranched covering. Furthermore all the defining polynomials of $W$ split in the local rings $\mathcal{O}_{u}$ for all points $u \in U^{\prime}$. If $V$ is a set in $\mathbb{C}^{n+k}$ then $V^{\prime}$ is defined to be $V^{\prime}:=V \cap \pi^{-1}\left(U^{\prime}\right)$ where $\pi: \mathbb{C}^{n+k} \rightarrow \mathbb{C}^{n}$ is the standard projection on the first $n$ variables. The structure of a $W$-type variety is studied in detail in [6].

Recall that $L$ is defined to be a common splitting field over $K$ of the polynomials $p_{1}, \ldots, p_{k}$. Without loss of generality one can assume that $L$ is contained in a fixed algebraic closure of $\mathcal{M}(U)$.

R e mark 2.3. Let $L$ be the common splitting field of the polynomials $p_{i}$. Take $u \in U^{\prime}$ (i.e. outside the discriminant variety) and put $\Omega:=\mathcal{M}_{u}$. Applying Lemma 2.2 we get a homomorphism $\sigma: L \rightarrow \mathcal{M}_{u}$ which maps the roots of the polynomials $p_{i}$ to germs of holomorphic functions.

The following simple observation on $K$-homomorphisms of $L$ to $\mathcal{M}_{u}$ will be used extensively.

Lemma 2.4. Let $L / K$ be a Galois extension and let $\sigma_{1}, \sigma_{2}: L \rightarrow \mathcal{M}_{u}$ be $K$-homomorphisms. Then there exists $g \in \operatorname{Gal}(L / K)$ such that $\sigma_{1}=\sigma_{2} \circ g$.

In the sequel we use the Riemann extension theorem to extend holomorphic functions through the discriminant locus. The assumptions of the theorem are satisfied due to the lemma:

Lemma 2.5 ([3], p. 86). Let $s \in \mathbb{C}$ be a root of a monic polynomial:

$$
s^{n}+a_{1} s^{n-1}+\ldots+a_{n}=0 .
$$

Then

$$
|s| \leq 2 \max _{i=1, \ldots, n}\left|a_{i}\right|^{1 / i}
$$

3. The covering $\operatorname{Hom}(L, \mathcal{M}) \rightarrow U^{\prime}$. In this section the set of all $K$ homomorphisms from $L$ to $\mathcal{M}_{u}$ is endowed with the structure of a covering of $U^{\prime}$, and is used, in the next section, to define a homomorphism of the fundamental group $\pi_{1}\left(U^{\prime}\right)$ into $\operatorname{Gal}(L / K)$.

Definition 3.1. Let $\operatorname{Hom}\left(L, \mathcal{M}_{u}\right)$ be the set of $K$-homomorphisms from $L$ to $\mathcal{M}_{u}$. We define $\operatorname{Hom}(L, \mathcal{M}):=\coprod_{u \in U^{\prime}} \operatorname{Hom}\left(L, \mathcal{M}_{u}\right)$ to be the disjoint $\operatorname{sum}$ of $\operatorname{Hom}\left(L, \mathcal{M}_{u}\right)$.

We introduce a topology on $\operatorname{Hom}(L, \mathcal{M})$ in a similar manner to the case of the sheaf of germs of holomorphic functions (cf. [4], p. 203).

Let $e_{1}, \ldots, e_{m}$ be a basis of the field extension $L / K$, and let $\sigma_{u} \in$ $\operatorname{Hom}(L, \mathcal{M})$ be a $K$-homomorphism from $L$ to $\mathcal{M}_{u}$. Let $\boldsymbol{f}_{i}:=\sigma_{u}\left(e_{i}\right)$ be the images of basis elements of $L$ in $\mathcal{M}_{u}$. Without loss of generality we can assume that the $\boldsymbol{f}_{i}$ 's are germs of holomorphic functions. Let the pairs 
$\left(U_{i}, f_{i}\right)$ for $i=1, \ldots, m$, where $f_{i} \in \mathcal{O}\left(U_{i}\right)$, be representatives of the germs $\boldsymbol{f}_{i}$ such that $U_{1} \cap \ldots \cap U_{m}$ is a connected open set. As the basis of the topology in $\operatorname{Hom}(L, \mathcal{M})$ we take all the sets of the form

$$
\left\{\sigma: e_{i} \mapsto\left(U_{i}, f_{i}\right)_{u^{\prime}}\right\}_{u^{\prime} \in U_{1} \cap \ldots \cap U_{m}}
$$

where $\left(U_{i}, f_{i}\right)_{u^{\prime}}$ denotes the germ of $f_{i}$ in the local ring $\mathcal{O}_{u^{\prime}}$.

Proposition 3.2. (1) The family $\mathcal{B}$ of all sets defined above is a basis of a topology.

$(2) \operatorname{Hom}(L, \mathcal{M})$ with the natural projection $\pi: \operatorname{Hom}(L, \mathcal{M}) \rightarrow U^{\prime}$ is a topological covering, i.e. for every $u \in U^{\prime}$ there exists an open, connected neighborhood $V$ of $u$ such that $\pi^{-1}(V)=\bigcup U_{\alpha}$ where $U_{\alpha}$ are disjoint open sets and $\left.\pi\right|_{U_{\alpha}}: U_{\alpha} \rightarrow V$ is a surjective homeomorphism.

Proof. The first part is obvious. We will give the proof of the second statement. Take $u \in U^{\prime}$. Let $\sigma_{u}$ be a $K$-homomorphism from $L$ to $\mathcal{M}_{u}$ (i.e. a point lying in the fiber of $u$ ). Let $\left\{\sigma_{u^{\prime}}: u^{\prime} \in V\right\}$ be an element of the basis of neighborhoods of $\sigma_{u}$. Then for every $g \in \operatorname{Gal}(L / K)$ the set $\left\{\sigma_{u^{\prime}} \circ g: u^{\prime} \in V\right\}$ is a neighborhood of $\sigma_{u} \circ g$ lying above $V$.

In this way we obtain the inclusion $\pi^{-1}(V) \supset \bigcup_{g \in \operatorname{Gal}(L / K)}\left\{\sigma_{u} \circ g\right\}$. The sets $\left\{\sigma_{u} \circ g\right\}$ are disjoint (by the identity principle) and open. It remains to show that $\pi^{-1}(V)=\bigcup_{g \in \operatorname{Gal}(L / K)}\left\{\sigma_{u} \circ g\right\}$. But by Lemma 2.4, given any two $K$-homomorphisms $\sigma_{1}, \sigma_{2}$ to $\mathcal{M}_{u_{0}}$, there is a $g \in \operatorname{Gal}(L / K)$ such that $\sigma_{1}=\sigma_{2} \circ g$. So for any $K$-homomorphism $\sigma$ lying above $u$ we can find an element of the Galois group $\operatorname{Gal}(L / K)$ such that $\sigma \in\left\{\sigma_{u^{\prime}} \circ g\right\}$.

Since $\operatorname{Hom}(L, \mathcal{M})$ is a covering, given a path $\gamma:[0,1] \rightarrow U^{\prime}$ and any $K$-homomorphism $\sigma_{0}$ from $L$ to $\mathcal{M}_{\gamma(0)}$, there is a unique lifting of $\gamma$ to a path $\widetilde{\gamma}:[0,1] \rightarrow \operatorname{Hom}(L, \mathcal{M})$ such that $\widetilde{\gamma}(0)=\sigma_{0}$. This lifting has good properties with respect to the action of $\operatorname{Gal}(L / K)$, namely we have

Proposition 3.3. Let $\gamma$ be a path in $U^{\prime}$, and let $\widetilde{\gamma}$ be its lifting to $\operatorname{Hom}(L, \mathcal{M})$ such that $\widetilde{\gamma}(0)=\sigma_{0}$. Then the map $t \mapsto(\widetilde{\gamma}(t)) \circ g$ is a lifting of $\gamma$ to $\operatorname{Hom}(L, \mathcal{M})$ starting from $\sigma_{0} \circ g$, for $g \in \operatorname{Gal}(L / K)$.

Furthermore, liftings of curves to $W^{\prime}$ can be constructed from a lifting to $\operatorname{Hom}(L, \mathcal{M})$. To define the $i$ th component of the path in $W^{\prime}$, first we lift $\gamma$ to $\operatorname{Hom}(L, \mathcal{M})$ obtaining a $K$-homomorphism from $L$ to $\mathcal{M}_{\gamma(t)}$. We can evaluate it on $s_{i}$ (a root of the polynomial $p_{i}$ ) to get a holomorphic function in the neighborhood of $\gamma(t)$. Finally, we take its value on $\gamma(t) \in U^{\prime} \subset \mathbb{C}^{n}$.

Proposition 3.4. If $\widetilde{\gamma}$ is a lifting of $\gamma$ to $\operatorname{Hom}(L, \mathcal{M})$, and $s_{i} \in X_{i}$, then the map

is a lifting of $\gamma$ to $W^{\prime}$.

$$
t \mapsto\left(\gamma(t),\left(\widetilde{\gamma}(t)\left(s_{1}\right)\right)(\gamma(t)), \ldots,\left(\widetilde{\gamma}(t)\left(s_{k}\right)\right)(\gamma(t))\right)
$$


We have two natural covering spaces of $U^{\prime}$, namely the original $W$-type variety $W^{\prime}$ and the covering $\operatorname{Hom}(L, \mathcal{M})$. The latter can be seen as encoding the algebraic relations between the coordinate functions (projections) restricted to $W^{\prime}$ :

$$
z_{i}: U \times \mathbb{C}^{k} \supset W^{\prime} \ni\left(u, z_{1}, \ldots, z_{k}\right) \mapsto z_{i} \in \mathbb{C} .
$$

EXAMPLE 3.5. If $W=\left\{z_{1}^{2}-u=0, \ldots, z_{k}^{2}-u=0\right\} \subset \mathbb{C} \times \mathbb{C}^{k}$ then $W^{\prime}$ is a $2^{k}$-sheeted covering of $U^{\prime} \subset \mathbb{C}$. The fiber over each point $u \in U^{\prime}$ of the covering $\operatorname{Hom}(L, \mathcal{M})$ is, by construction, bijective with the Galois group $\operatorname{Gal}(L / K)$. In this case $L$ is the splitting field of the polynomial $z^{2}-u$ over $\mathbb{C}(u)$. So the Galois group $\operatorname{Gal}(L / K)$ is $\mathbb{Z}_{2}$. The covering $\operatorname{Hom}(L, \mathcal{M})$ is therefore 2-sheeted. We see therefore that in general $W^{\prime}$ is not isomorphic to $\operatorname{Hom}(L, \mathcal{M})$.

EXAMPLE 3.6. If $W=\left\{z_{1}^{2}-u_{1}=0, \ldots, z_{k}^{2}-u_{k}=0\right\} \subset \mathbb{C}^{k} \times \mathbb{C}^{k}$ then $W^{\prime}$ is also a $2^{k}$-sheeted covering of $U^{\prime} \subset \mathbb{C}^{k}$. But now $L$ is the splitting field of the polynomial $\left(z^{2}-u_{1}\right) \cdot \ldots \cdot\left(z^{2}-u_{k}\right)$ over the field $\mathbb{C}\left(u_{1}, \ldots, u_{k}\right)$. The Galois group is now $\mathbb{Z}_{2} \times \ldots \times \mathbb{Z}_{2}$. Here the covering $\operatorname{Hom}(L, \mathcal{M})$ is also $2^{k}$-sheeted, and one can show that it is in fact isomorphic to $W^{\prime}$.

In some cases the structure of $\operatorname{Hom}(L, \mathcal{M})$ can be richer than that of the original variety $W^{\prime}$.

ExAmple 3.7. Let $W=\left\{z^{n}+u_{1} z^{n-1}+\ldots+u_{n}=0\right\}$. Hence $W^{\prime}$ is a $n$-sheeted covering. But now the Galois group $\operatorname{Gal}(L / K)$ is isomorphic to the permutation group $S_{n}$. Therefore $\operatorname{Hom}(L, \mathcal{M})$ is a $n !$-sheeted covering of $U^{\prime}$.

4. Homomorphism $\pi_{1}\left(U^{\prime}\right) \rightarrow \operatorname{Gal}(L / K)$. In this section we define a homomorphism of the fundamental group of $U^{\prime}$ to the $\operatorname{Galois} \operatorname{group} \operatorname{Gal}(L / K)$ and establish some of its properties.

Let $\sigma_{0}: L \rightarrow \mathcal{M}_{u_{0}}$ be a $K$-homomorphism, and $\gamma$ be some representative of $[\gamma] \in \pi_{1}\left(U^{\prime}, u_{0}\right)$. There is a unique lifting of $\gamma$ to $\operatorname{Hom}(L, \mathcal{M})$ starting from $\sigma_{0}$. Since $\gamma$ is a closed loop, $\widetilde{\gamma}(1)$ is also a $K$-homomorphism from $L$ to $\mathcal{M}_{u_{0}}$. Then by Lemma 2.4 there is a unique $g \in \operatorname{Gal}(L / K)$ such that $\widetilde{\gamma}(1)=\sigma_{0} \circ g$. By the homotopy lifting property of coverings, $g$ is independent of the choice of a representative of $[\gamma] \in \pi_{1}\left(U^{\prime}, u_{0}\right)$.

Definition 4.1. We shall denote by $\mathfrak{G a l}$ the mapping of the fundamental group $\pi_{1}\left(U^{\prime}, u_{0}\right)$ to the Galois group $\operatorname{Gal}(L / K)$ obtained by the above construction.

Proposition 4.2. Let $\gamma_{1}, \gamma_{2} \in \pi_{1}\left(U^{\prime}, u_{0}\right)$. Then $\mathfrak{G a l}\left(\gamma_{2} \circ \gamma_{1}\right)=\mathfrak{G a l}\left(\gamma_{2}\right) \circ$ $\mathfrak{G} \mathfrak{a l}\left(\gamma_{1}\right)$. 
Proposition 4.3. (1) If $K=\mathbb{C}(u)$ then $\mathfrak{G a l}\left(\pi_{1}\left(U^{\prime}\right)\right)=\operatorname{Gal}(L /(L \cap$ $\widetilde{\mathcal{N}}(U))$ ) (here $\tilde{\mathcal{N}}(U)$ denotes the field of fractions of $\mathcal{N}(U)$ ).

(2) In the analytic or Nash case $(R=\mathcal{O}(U)$ or $R=\mathcal{N}(U))$, the mapping $\mathfrak{G a l}$ is an epimorphism.

Proof. Take an element $f \in L$ invariant with respect to $\mathfrak{G a l}\left(\pi_{1}\left(U^{\prime}\right)\right)$. Since $L$ is algebraic over $K$ we have $L=K\left(s_{1}, \ldots, s_{p}\right)=K\left[s_{1}, \ldots, s_{p}\right]$, where $s_{i}$ are the roots of the defining polynomials of $W$. Therefore we can write $f=\sum_{j=1}^{p} w_{j} t_{j}$, where $w_{j} \in K$ and $t_{j}$ are monomials in $s_{1}, \ldots, s_{p}$. Multiplying by the denominators of $w_{j}$ we get $F:=h f=\sum_{j=1}^{p} v_{j} t_{j}$, where $h, v_{j} \in R$ ( $K$ is the field of fractions of $R$ ).

By Remark 2.3 we have $\sigma_{u}(F) \in \mathcal{O}_{u}$ for every $u \in U^{\prime}$. We define $\widehat{F}$ : $U^{\prime} \rightarrow \mathbb{C}$ in the following way:

$$
\widehat{F}(u):=\sigma_{u}(F)(u)
$$

where $\sigma_{u}$ is obtained from $\sigma_{0}$ by a lifting along some path. It is defined only up to the action of $g \in \mathfrak{G} \mathfrak{a l}\left(\pi_{1}\left(U^{\prime}\right)\right)$, but since $F$ is $\mathfrak{G a l}\left(\pi_{1}\left(U^{\prime}\right)\right)$-invariant the images of $F$ in $\mathcal{O}_{u}$ coincide.

$\widehat{F}$ is clearly holomorphic in $U^{\prime}$, and locally bounded in $U$. By the Riemann extension theorem it can be extended to a holomorphic function in $U$. Now $\widehat{F}=F$ since $\sigma_{u_{0}}$ is a monomorphism. This shows that $f=F / h$ is an element of $\mathcal{M}(U)$.

Conversely, if $f \in L$ is holomorphic then it is $\mathfrak{G} \mathfrak{a l}\left(\pi_{1}\left(U^{\prime}\right)\right)$-invariant.

5. Algebraic set associated with an orbit of $\operatorname{Gal}(L / K)$. Recall that $L$ is the common splitting field of the defining polynomials $p_{1}, \ldots, p_{k}$ of $W$. Let $X_{i} \subset L$ be the zero-set of $p_{i}$ in $L$. The group $\operatorname{Gal}(L / K)$ acts in a natural way on $X_{1} \times \ldots \times X_{k} \subset L^{k}$. An orbit is therefore a finite set of points in $L^{k}$. Since $L$ is an infinite field there exists a collection of polynomials in $k$ variables whose common zeroes are precisely the given finite set of points.

In the sequel we shall use a standard choice of these polynomials, called the canonical equations.

Definition 5.1. ([6], Appendix V, p. 369, Canonical equations). Let $S:=\left\{\left(s_{1}^{(j)}, \ldots, s_{k}^{(j)}\right)\right\}_{j=1, \ldots, m} \subset L^{k}$ be a set of $m$ points. For every $m$-tuple $\mu=\left(\mu_{1}, \ldots, \mu_{m}\right) \in \mathbb{N}^{m}$ such that $|\mu|=\mu_{1}+\ldots+\mu_{m}=m$, the polynomial $\Phi_{\mu} \in L\left[z_{1}, \ldots, z_{k}\right]$ is defined by

$$
\Phi_{\mu}(z)=\sum_{\nu}^{(\mu)}\left(z_{\nu_{1}}-s_{\nu_{1}}^{(1)}\right) \cdot \ldots \cdot\left(z_{\nu_{m}}-s_{\nu_{m}}^{(m)}\right)
$$

where the summation is over all $m$-tuples $\nu$ such that every $j \in\{1, \ldots, k\}$ appears exactly $\mu_{j}$ times in $\left(\nu_{1}, \ldots, \nu_{k}\right)$. The set of common zeroes of the above defined polynomials is precisely the set $S$. 
It is easy to see that the polynomials $\Phi_{\mu}$ have coefficients in $K$ when $S$ is taken to be $\operatorname{Gal}(L / K)$-invariant. In fact, we shall use a more precise result.

Proposition 5.2. If $K=\mathbb{C}(u)$ then $\Phi_{\mu} \in \mathbb{C}[u]\left[z_{1}, \ldots, z_{k}\right]$.

Proof. For every irreducible polynomial $P \in \mathbb{C}[u]$ one can define a valuation $\nu_{P}: \mathbb{C}(u)-\{0\} \rightarrow \mathbb{Z}$ by

$$
f=P^{\nu_{P}(f)} \cdot \frac{Q}{R}, \quad f \in \mathbb{C}(u)-\{0\},
$$

where $P, Q$ and $R$ are relatively prime (cf. [1], p. 139). Since $L / \mathbb{C}(u)$ is algebraic, one can extend this valuation to the field $L$ (see [1], p. 144).

Let $s \in L$ be a zero of one of the defining polynomials of $W$. We will show that $\nu_{P}(s) \geq 0$. Suppose that $\nu_{P}(s)<0$; then, since $s^{n}+\sum_{i} a_{i} s^{i}=0$, we have

$$
n \nu_{P}(s)=\nu_{P}\left(-\sum_{i} a_{i} s^{i}\right) \geq \min _{i=0, \ldots, n-1}\left(\nu_{P}\left(a_{i}\right)+i \nu_{P}(s)\right)>n \nu_{P}(s) .
$$

This is a contradiction. In the last inequality we used the fact that, since the $a_{i}$ are polynomials, $\nu_{P}\left(a_{i}\right) \geq 0$.

The coefficients of the polynomials $\Phi_{\mu}$ are linear combinations of monomials in $s$, therefore $\nu_{P}$ (coefficients) $\geq 0$. The coefficients are elements of $\mathbb{C}(u)$, so the nonnegativity of $\nu_{P}$ for all irreducible polynomials $P$ implies that the coefficients lie in $\mathbb{C}[u]$.

Since the $s_{i}$ are mapped locally to holomorphic functions in $U^{\prime}$ (locally bounded in $U$ ) by $K$-homomorphisms $\sigma \in \operatorname{Hom}(L, \mathcal{M})$, the coefficients are holomorphic in $U^{\prime}$ and can be extended by the Riemann extension theorem to the whole of $U$. Thus the $\Phi_{\mu}$ always define an analytic set.

Definition 5.3. Let $S \subset X_{1} \times \ldots \times X_{k}$ be an orbit of $\operatorname{Gal}(L / K)$, consisting of $m$ points. Define

$$
V_{S}:=\left\{(u, z) \in U \times \mathbb{C}^{k}\left|\Phi_{\mu}(u, z)=0,\right| \mu \mid=m\right\},
$$

where $\Phi_{\mu}$ are the canonical equations of $S$.

LEMMA 5.4. The algebraic (resp. analytic, Nash) variety $V_{S}$ has the following properties:

1. $V_{S}^{\prime}:=\pi^{-1}\left(U^{\prime}\right) \cap V_{S}$ is a covering of $U^{\prime}$ and $V_{S}^{\prime} \subset W$,

2. $V_{S}^{\prime}$ is connected,

3. $V_{S}=\overline{V_{S}^{\prime}} \subset W$.

Proof. (1) Choose $u \in U^{\prime}$ and a $K$-homomorphism $\sigma_{0}: L \rightarrow \mathcal{M}_{u}$. Since the $s_{i} \in S$ are mapped by $\sigma_{0}$ to holomorphic functions in a neighborhood 
of $u$, one can evaluate them at $u$. Taking $\widehat{S}$ to be the resulting set of points in $\mathbb{C}^{k}$ and repeating the construction of the polynomials $\Phi_{\mu}$ one obtains $\pi^{-1}(u) \cap V_{S}=\widehat{S}$. It follows immediately that $V_{S}^{\prime} \subset W^{\prime}$, and that it is a subcovering.

(2) Let $u_{0} \in U^{\prime}$ and $\sigma_{0}: L \rightarrow \mathcal{M}_{u_{0}}$ be as in the definition of the homomorphism $\mathfrak{G a l}$. We will show that any two points $P=\left(u, z_{1}, \ldots, z_{k}\right) \in$ $V_{S}^{\prime}$ and $P^{\prime}=\left(u^{\prime}, z_{1}^{\prime}, \ldots, z_{k}^{\prime}\right) \in V_{S}^{\prime}$ can be connected by a path in $V_{S}^{\prime}$. Without loss of generality one can assume that $u=u_{0}$.

(a) Take $u^{\prime}=u=u_{0}$. From the proof of (1) one can find $\left(s_{1}, \ldots, s_{k}\right)$, $\left(s_{1}^{\prime}, \ldots, s_{k}^{\prime}\right) \in S \subset X_{1} \times \ldots \times X_{k}$ which map by $\sigma_{0}$ to the points $P$ and $P^{\prime}$. There is a $g \in \operatorname{Gal}(L / K)$ which takes $\left(s_{1}, \ldots, s_{k}\right)$ to $\left(s_{1}^{\prime}, \ldots, s_{k}^{\prime}\right)$. There is a corresponding loop $[\gamma] \in \pi_{1}\left(U^{\prime}, u_{0}\right)$ which maps to $g$. Proposition 3.4 gives a lifting of $\gamma$ to $V_{S}^{\prime} \subset W$ starting from $P$ and ending at $P^{\prime}$.

(b) General case $u^{\prime} \neq u=u_{0}$. Transporting $\sigma_{0}$ from $u_{0}$ to $u^{\prime}$ gives a $K$ homomorphism $\sigma: L \rightarrow \mathcal{M}_{u^{\prime}}$. One can find $\left(s_{1}^{\prime}, \ldots, s_{k}^{\prime}\right) \in S \subset X_{1} \times \ldots \times X_{k}$ which $\sigma$ maps to $P^{\prime}$. Using Proposition 3.4 again, we obtain a path in $V_{S}^{\prime}$ joining $P^{\prime}$ and some point in the fiber over $u_{0}$. This reduces the proof to case (a).

(3) The inclusion $V_{S} \supset \overline{V_{S}^{\prime}}$ is obvious. Take $u \in \Delta$. We construct the set $\widehat{S} \subset \mathbb{C}^{k}$ in the following way. Take $u_{0} \in U^{\prime}, \sigma_{0}: L \rightarrow \mathcal{M}_{u_{0}}$ and $\left(s_{1}, \ldots, s_{k}\right)$ $\in S$. For some path $\gamma:[0,1] \rightarrow U$ such that $\gamma(0)=u_{0}, \gamma(1)=u$ and $\gamma([0,1))$ $\subset U^{\prime}$ define

$$
\widehat{s}_{i}:=\lim _{x \rightarrow 1}(\widetilde{\gamma}(x))\left(s_{i}\right)(\gamma(x))
$$

where $\widetilde{\gamma}$ is a lifting of $\gamma$ to $\operatorname{Hom}(L, \mathcal{M})$ starting from $\sigma_{0}$.

The expression $(\widetilde{\gamma}(x))\left(s_{i}\right)(\gamma(x))$ in the above limit is a lifting of $\gamma$ to $W$ projected onto the $i$ th variable. Since $W$ is bounded there is an accumulation point. Since $W$ is closed there are at most a finite number of such points. But since $(\widetilde{\gamma}(x))\left(s_{i}\right)(\gamma(x))$ is a continuous function in $x$, the limit exists.

Repeating the construction of $\Phi_{\mu}$ using the set $\widehat{S}$ gives $\pi^{-1}(u) \cap V_{S} \subset$ $\widehat{S} \subset \overline{V_{S}^{\prime}}$.

To obtain the inclusion $V_{S} \subset W$ note that since $V_{S}^{\prime} \subset W^{\prime}$, we have $V_{S}=\overline{V_{S}^{\prime}} \subset \overline{W^{\prime}}=W$.

6. The main theorem. Now, we are in a position to prove our main theorem.

Let us recall the following notation and basic definitions. An algebraic (resp. Nash, analytic) $W$-type variety in a connected open set $U \times \mathbb{C}^{k} \subset \mathbb{C}^{n+k}$ is a set of the form

$$
W=\left\{\left(u, z_{1}, \ldots, z_{k}\right) \in U \times \mathbb{C}^{k} \mid p_{i}(u)\left(z_{i}\right)=0, i=1, \ldots, k\right\}
$$


where

$$
p_{i}(u)(z)=z^{n_{i}}+\sum_{j=0}^{n_{i}-1} a_{j}^{i} z^{j} \in R[z], \quad a_{j}^{i} \in R .
$$

Here $R$ denotes the ring $\mathbb{C}[u]$ (resp. $\mathcal{N}(U), \mathcal{O}(U)$ ), and $K$ the field of fractions of $R$. $L$ is the common splitting field of the polynomials $p_{1}, \ldots, p_{k}$ over $K$. And finally $X_{i} \subset L$ is the zero-set of the polynomial $p_{i} \in R[z]$ in $L$.

THEOREM 6.1. Suppose that one of the following holds:

1. $W$ is a $W$-type algebraic variety in $\mathbb{C}^{n} \times \mathbb{C}^{k}$,

2. $W$ is a $W$-type Nash variety in a connected open set $U \times \mathbb{C}^{k} \subset \mathbb{C}^{n+k}$,

3. $W$ is a $W$-type analytic variety in a connected open set $U \times \mathbb{C}^{k} \subset \mathbb{C}^{n+k}$.

Then the irreducible components of $W$ are in 1:1 correspondence with the orbits of $\operatorname{Gal}(L / K)$ in the set $X_{1} \times \ldots \times X_{k}$.

Proof. Let $V_{S}=\left\{\Phi_{\mu}=0\right\}$ be the set associated with an orbit as in Definition 5.3. Then by Lemma 5.4, $V_{S}=\overline{V_{S}^{\prime}}$ where $V_{S}^{\prime}=\pi^{-1}\left(U^{\prime}\right) \cap V_{S}$. Since $V_{S}^{\prime}$ is a connected submanifold (Lemma 5.4), $V_{S}$ is irreducible. Here we have used the simple observation that $V_{S}^{\prime}$ is dense in the regular part of $V_{S}$.

Let $V$ be an irreducible component of $W$. Then by ([3], p. 215), $V$ is the closure of a connected component of the regular part $\operatorname{Reg} W$ of $W$, $V=\bar{Z}$. Let $(u, z) \in Z^{\prime}$. Then $(u, z) \in V_{S}$ for some orbit of $\operatorname{Gal}(L / K)$. By Lemma 5.4, $V_{S}^{\prime}$ is connected, so $V_{S}^{\prime} \subset Z \subset V$. Taking closures, and using the fact that $\overline{V_{S}^{\prime}}=V_{S}$, we obtain $\overline{V_{S}} \subset \bar{Z}=V$.

Since $\operatorname{dim} V_{S}=\operatorname{dim} V$, the inclusion is indeed an equality.

Acknowledgements. I would like to express my gratitude to Dr. Piotr Tworzewski who led me to consider this problem. I would like to thank Dr. Sławomir Cynk for many helpful discussions.

\section{References}

[1] S. Balcerzyk and T. Józefiak, Commutative Noetherian and Krull Rings, PWN, Warszawa, and Ellis Horwood, Chichester, 1989.

[2] N. Jacobson, Lectures in Abstract Algebra, Vol. III, Grad. Texts in Math. 32, Springer, 1964.

[3] S. Łojasiewicz, Introduction to Complex Analytic Geometry, Birkhäuser, 1991.

[4] S. G. Krantz, Function Theory of Several Complex Variables, Wiley, New York, 1982 . 
[5] P. Tworzewski, Intersections of analytic sets with linear subspaces, Ann. Scuola Norm. Sup. Pisa 17 (1990), 227-271.

[6] H. Whitney, Complex Analytic Varieties, Addison-Wesley, 1972.

Institute of Physics

Jagiellonian University

Reymonta 4

30-059 Kraków, Poland

E-mail: ufrjanik@jetta.if.uj.edu.pl

Reçu par la Rédaction le 20.5.1996

Révisé le 30.8.1996 